\title{
Reconciling Tap-Changing Transformer Models
}

\author{
Jose M. Cano, Member, IEEE, Md Rejwanur R. Mojumdar, and Gonzalo A. Orcajo, Member, IEEE
}

\begin{abstract}
The model of the tap changing transformer used in classic power system studies, including load flow analysis or state estimation, is still somehow controversial. Two alternative formulations can be found in the literature, which have been adopted by the most important software packages. This work demonstrates that those formulations lead to similar results near the principal tap but to important discrepancies at extreme tap positions, with different impact depending on the power factor of the power flowing through the transformer. Moreover, a general model that fully explains those differences is proposed. The new model allows to adopt a third alternative that, without requiring further data than those used by traditional formulations, leads to highly improved results.
\end{abstract}

Index Terms-Power transformers, tap changers, transformer models.

\section{INTRODUCTION}

$\mathbf{T}$ HE limited amount of information generally available about transformers, and specifically about tap-changing transformers, leads to the fact that a quite simplified model of these devices is used in such usual tasks as load flow analysis or state estimation. The data is obtained from the nameplate of the device and comprises the rated power and voltage values, short-circuit impedance and tap positions. Only transformers with a tapping range exceeding $\pm 5 \%$ are obliged by standards to provide further information about the shortcircuit impedance (at least, values for the extreme tappings are required in that case) [1].

Two different tap-changing transformer models can be found today in both the literature and practical software implementations [2]-[4]. However, these two models yield different results, which can easily mislead the user in certain tasks, as during the validation of engineering or research results with an external tool. While these discrepancies can be considered merely trivial at the principal tap, this work demonstrates that the inconsistency can lead to huge differences at extreme tap positions. This fact was previously observed by other authors [5] but, they chose one of the alternatives and focused their efforts on the manipulation of the other model to reach the same results. On the contrary, this work explains the causes of those discrepancies and proposes a reconciled solution.

In section II, a new model for the tap-changing transformer is proposed, which opens the door to a much more accurate description of the device. Section III uses the new model to clearly explain the reasons for the aforementioned discrepancies. An assessment of the errors caused by traditional models is presented in section IV. Section V describes a case study

Jose M. Cano and Gonzalo A. Orcajo are with the Department of Electrical Engineering, University of Oviedo, Gijón, Asturias, 30013 Spain, e-mail: jmcano@uniovi.es, gonzalo@uniovi.es

Md Rejwanur R. Mojumdar is with the Department of Energy and Petroleum Engineering, University of Stavanger, 4036 Stavanger, Norway, email: md.r.mojumdar@uis.no.

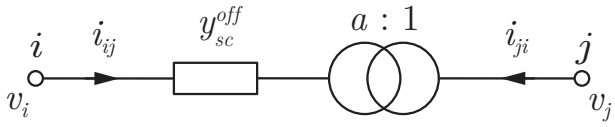

Fig. 1. Model of the tap-changing transformer with short-circuit impedance at the off-nominal turns side

to highlight the importance of the new proposal. Finally, the conclusions of this study are drawn in section VI.

\section{Description of the New Model}

Power system studies do not normally require the shunt branch of the equivalent model of the transformer to be taken into account. Thus, when dealing with nominal turns ratios, the use of the detailed or simplified model of the transformer makes no difference, as in both cases the device is reduced to a series impedance. However, this is no longer true when the transformer is using an off-nominal turns ratio. As it is demonstrated in this work, the simplified model of the transformer can lead to important errors.

Let us consider a transformer with off-nominal turns ratio $a: 1$ as depicted in Fig. 1. Although the following parameters are not normally known by the user, let $k$ be the ratio between the p.u. impedance in the nominal winding, $z_{2}$, and tapped winding, $z_{1}$, (for the sake of simplicity, the same ratio is considered for resistance and leakage reactance). From the offnominal side, the series admittance can then be calculated as

$$
y_{s c}^{o f f}=\frac{1}{z_{1}+a^{2} z_{2}}=\frac{1+k}{1+k a^{2}} y_{s c},
$$

$y_{s c}$ being the short-circuit admittance obtained during the short-circuit test at the principal tap (normally shown at the nameplate of the device as an impedance, $z_{s c}$ ), or at the current tap position, if further data are available.

The application of Kirchhoff Laws and the well-known relationships that apply to the ideal transformer yields

$$
\begin{gathered}
v_{i}=\frac{i_{i j}}{y_{s c}^{o f f}}+a v_{j}, \\
i_{i j}=-\frac{i_{j i}}{a},
\end{gathered}
$$

and thus, the nodal equations of the device can be written as

$$
\left[\begin{array}{c}
i_{i j} \\
i_{j i}
\end{array}\right]=\left[\begin{array}{cc}
Y_{i i} & Y_{i j} \\
Y_{j i} & Y_{j j}
\end{array}\right]\left[\begin{array}{c}
v_{i} \\
v_{j}
\end{array}\right]
$$

where

$$
\begin{gathered}
Y_{i i}=\frac{1+k}{1+k a^{2}} y_{s c}, \\
Y_{i j}=Y_{j i}=-\frac{a(1+k)}{1+k a^{2}} y_{s c}, \\
Y_{j j}=\frac{a^{2}(1+k)}{1+k a^{2}} y_{s c .}
\end{gathered}
$$




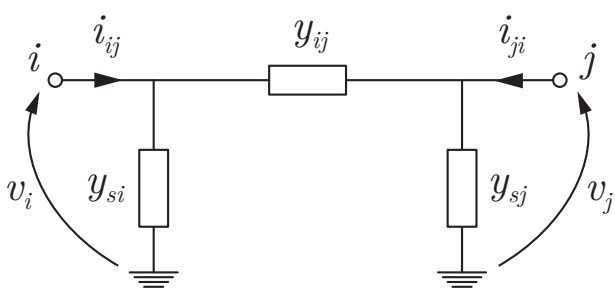

Fig. 2. $\pi$ equivalent model of the tap-changing transformer
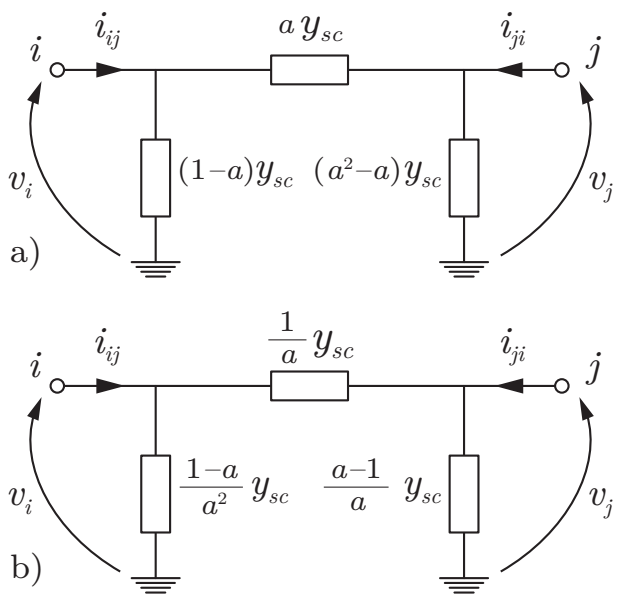

Fig. 3. $\pi$ equivalent traditional models of tap-changing transformers

Hence, the components of the $\pi$ equivalent of the new model, shown in Fig. 2, can be calculated as

$$
\begin{gathered}
y_{i j}=-Y_{i j}=\frac{a(1+k)}{1+a^{2} k} y_{s c}, \\
y_{s i}=Y_{i i}+Y_{i j}=\frac{1-a+k(1-a)}{1+a^{2} k} y_{s c}, \\
y_{s j}=Y_{j j}+Y_{i j}=\frac{a(a-1)(1+k)}{1+a^{2} k} y_{s c} .
\end{gathered}
$$

\section{ReConciliation of Previous Models}

As it is easily proved, the two well-known models, extensively used in the literature and practical implementations, correspond to the particular cases of making the parameter $k$ equal to 0 and $\infty$ in (1), and thus in (8)-(10). In particular, $k=0$ corresponds to the option of considering all the shortcircuit impedance of the transformer as being provided by the winding at the off-nominal turns side. In this case, the off-nominal admittance of the transformer, $y_{s c}^{o f f}$, turns to be the same as $y_{s c}$, and the well-known parameters of the $\pi$ equivalent model shown in Fig. 3(a) are obtained. On the other hand, $k=\infty$ corresponds to the option of considering all the short-circuit impedance of the transformer as being provided by the winding at the nominal turns side. In this case, the off-nominal admittance of the transformer, $y_{s c}^{o f f}$, turns to be $y_{s c} / a^{2}$, and the set of parameters of the $\pi$ equivalent model shown in Fig. 3(b) is reached. It is important to highlight that the new model opens the door to obtain accurate results if $k$ is provided by the manufacturer. But, even if this is not the case, much more realistic estimates can be obtained if $k$ is set to 1 , which is a common engineering practice adopted when the detailed model of the transformer is to be used [6], [7].

Finally, notice that in [5], the authors pointed out the inconsistency of the two alternative models, and concluded that, in order to make them yield the same results the admittance in Fig. 3(a) should be previously affected by $1 / a^{2}$. While this is a pertinent observation, this approach does not solve the fact that all the short-circuit admittance is being assigned to the nominal winding, which is far from being realistic and can lead to important errors.

\section{ERROR ASSESSMENT}

For a given value of the off-nominal turns side variables, $v_{i}$ and $i_{i}$, the new model allows the calculation of the nominal turns side voltage $v_{j}$. If the result, for a generic value of $k, v_{j}^{k}$, is taken as a reference, and the calculation is repeated for the values used by traditional models, thus obtaining $v_{j}^{0}$ and $v_{j}^{\infty}$, an assessment of the mismatch voltage can be obtained from (8), (9) and (10), which represent the error of these popular approaches,

$$
\begin{aligned}
& \Delta v_{j}^{0}=v_{j}^{0}-v_{j}^{k}=\frac{k\left(1-a^{2}\right)}{a(1+k)} \frac{i_{i}}{y_{s c}}, \\
& \Delta v_{j}^{\infty}=v_{j}^{\infty}-v_{j}^{k}=\frac{a^{2}-1}{a(1+k)} \frac{i_{i}}{y_{s c}} .
\end{aligned}
$$

\section{CAse Study}

In order to highlight the important differences that can arise when using the traditional transformer models depicted in Fig. 3 and the benefits of the newly proposed formulation, a case study is presented in this section. Let us consider an $80 \mathrm{MVA}, 50 \mathrm{~Hz}, 220 / 132 \mathrm{kV} \pm 10 \%$ transformer with a nameplate short-circuit impedance, $z_{s c}$, of $0.01+0.12 j$ and a tap changer, located on the highest voltage side, with 21 positions and a tapping step of $1 \%$. If further data about the short-circuit impedance at extreme tap positions were available, as it should be according to [1], a different value of $z_{s c}$ could be calculated by linear interpolation for any tap position. In any case, this straightforward task will not be used in this case study not to obscure the core of the proposal.

Fig. 4 shows the voltage of the transformer at the nominal turns side when fed by a constant voltage of 1 pu at the offnominal turns side for each tap position available. In each case, the transformer is delivering the rated current at the offnominal turns side. Two different power factors are considered by selecting the phase angle between $v_{i}$ and $i_{i j}$, which is called $\theta$ in the following: (a) a unity power factor, $\theta=0^{\circ}$, and (b) a pure capacitive case, $\theta=90^{\circ}$. The voltage is calculated both for the traditional models $(k=0$ and $k=\infty)$ and for the proposed model, assuming a fair contribution of both windings to the short-circuit impedance, i.e. $k=1$. Although this assumption is probably not exact (this data is seldom provided by the manufacturer), it is for sure a better estimate in line with accepted engineering practices. In Fig. 4.(a) the module of the voltage at the off-nominal turns side is shown. At high power factors, the differences between the alternative transformer 


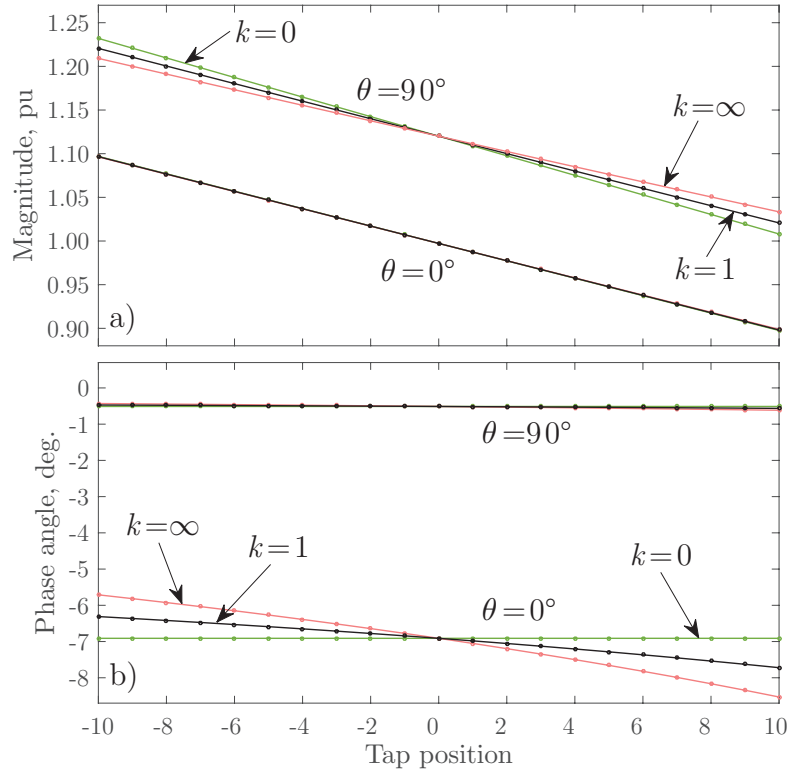

Fig. 4. Nominal-turns side voltage for the different tap positions. Transformer at rated current with two different power factors: unity $\left(\theta=0^{\circ}\right)$ and pure capacitive $\left(\theta=90^{\circ}\right)$. (a) Voltage magnitude, and (b) voltage phase angle

models can be ignored. However, important discrepancies arise at poor power factors. On the other hand, Fig. 4.(b) shows the phase angle of the voltage at the off-nominal turns side. The differences between the different models result evident now at high power factors. The new model offers a consensus estimate even if $k$ is not accurately known.

The errors arisen from the use of traditional models, calculated by taking the new model as a reference (with $k=1$ ), were obtained at rated current for every tap position and power factor (including reverse power flow). In Fig. 5.(a) the maximum deviation of the voltage at the nominal turns side, $\left|v_{j}^{0}\right|-\left|v_{j}^{1}\right|$ and $\left|v_{j}^{\infty}\right|-\left|v_{j}^{1}\right|$, is depicted for each power factor. This graph proves that the error in the calculation of the voltage can rise to near $1.3 \%$ in extreme positions of the tap changer when dealing with poor power factors. Notice that the same result can be obtain from (11) and (12). Fig. 5.(b) shows the maximum deviation of the phase angle of the voltage at the nominal turns side. Noticeably, this error evolves in the opposite direction, being maximum for high power factors, when it reaches values as high as $0.8^{\circ}$, and negligible for reactive power flows. In a symmetrical tap changer, as the one considered in this work, the maximum errors are found at the highest position of the tap (and not at the lowest), as can be easily proved from (11) and (12).

\section{CONCLUSION}

The use of the simplified equivalent model of the transformer is universally admitted when conducting power system studies, due to the low impact of the magnetizing branch and the inherent benefit of removing a useless bus from the problem. However, neglecting the fact that the short-circuit impedance is the result of contributions from two different windings can lead to unacceptable errors in the formulation of the tap-changing transformer model. This work proposes a new general model which includes the contribution of each

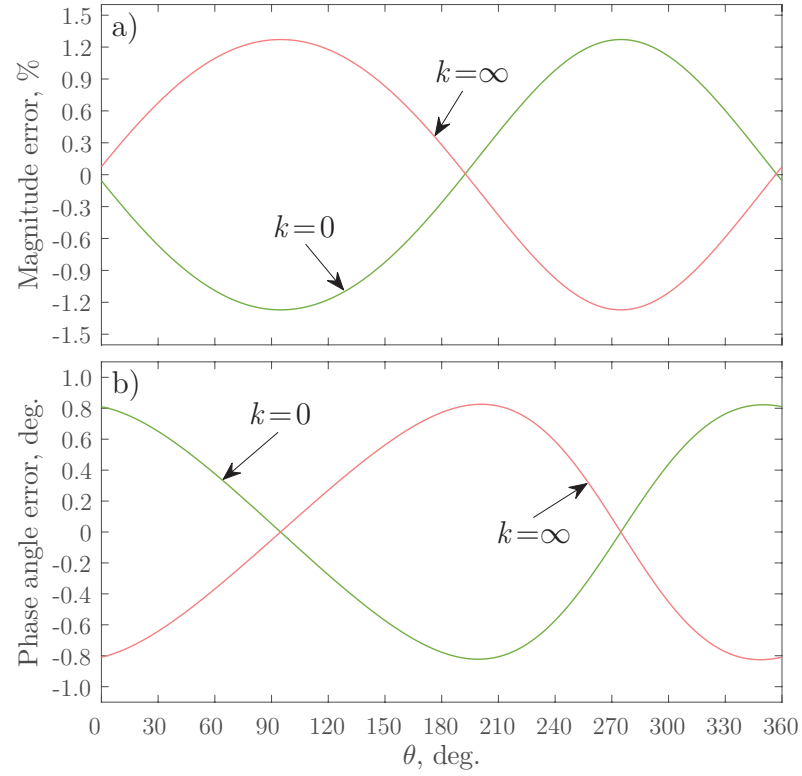

Fig. 5. Maximum deviation in the calculation of the nominal-turns side voltage at rated current. (a) Voltage magnitude, and (b) voltage phase angle

winding to the short-circuit impedance. Although this data is not generally available, the new model allows to consider a fair contribution (50/50) of both windings to this parameter, which is an accepted practice in engineering. The new model can be tuned to match the results from traditional alternatives, which consider the short-circuit impedance as caused by only one of the transformer windings, either the off-nominal or nominal turns side. This fact makes the new model useful to understand the basis of each formulation, providing a clear perspective on the influence of the underlying assumptions. The work demonstrates that the errors caused by traditional models can be unacceptable at extreme tap positions and are greatly influenced by the operating point of the transformer. The inclusion of the proposed tap-changing transformer model in power system software packages, tuned with the recommended values shown in this work, can significantly help to improve the accuracy of power system studies without the need to request additional data from the user.

\section{ACKNOWLEDGMENT}

This work was supported by the Spanish Government Innovation Development and Research Office (MEC) under research Grant DPI2017-89186-R.

\section{REFERENCES}

[1] Power transformers - Part 1: General, IEC 60076-1:2011, IEC Std., 2011.

[2] J. D. Glover, T. J. Overbye, and M. S. Sarma, Power System Analysis and Design, 6th ed. Andover, UK: Cengage Learning, Inc., Jul. 2016.

[3] PowerWorld User's Guide, PowerWorld Corporation, 2011, [Online]. Available: http://www.powerworld.com.

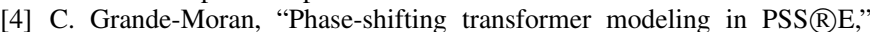
Siemens Power Technology, vol. 1, no. 111, pp. 1-7, Mar. 2012.

[5] L. V. Barboza, H. H. Zürn, and R. Salgado, "Load tap change transformers: A modeling reminder," IEEE Power Engineering Review, vol. 21, no. 2, pp. 51-52, Feb. 2001.

[6] S.V.Kulkarni and S.A.Khaparde, Transformer Engineering: Design, Technology and Diagnostics, 2nd ed. Boca Raton: CRC Press, May 2013.

[7] K. Shaarbafi, "Transformer modelling guide," Alberta Electric System Operator, Tech. Rep., Jul. 2014. 